\title{
New roles for astrocytes: Regulation of CNS synaptogenesis
}

\author{
Michal Slezak and Frank W. Pfrieger
}

Max-Planck/CNRS Group, UPR 2356, Centre de Neurochimie, 5 rue Blaise Pascal, F-67084 Strasbourg, France

The notion that astrocytes have a profound influence on the function of synapses between CNS neurons implies that the development of synaptic connections and their glial neighbors are controlled by reciprocally acting signals. Currently, however, synaptogenesis is considered a purely neuronal affair. This article summarizes recent experimental evidence suggesting that this may not be the case. Astrocytes may indeed regulate the formation, maturation and maintenance of synapses. The recent advances caution that synapses cannot develop correctly without astrocytes. Further progress on this issue requires new experimental models to identify signaling pathways and to scrutinize the relevance of glia-synapse interactions in vivo.

During the past decade, an ageing star has reappeared on the CNS stage, playing a new role in an old piece that before featured only two actors. That star is the astrocyte and the play is the function of synaptic connections between two partner neurons [1-5]. This new triad of actors has prompted the concept of the 'tripartite synapse', which states that 'synaptically associated astrocytes should be viewed as integral modulatory elements of synapses' [6]. However, recent reviews on the development of synapses [7-13] reveal that this fundamental process is still considered an entirely neuronal event. This article summarizes evidence that this view could be outdated and that astrocytes might take part in this process as well. As is true for all liaisons, the life of a synaptic connection between two partner neurons can be divided into three phases: (i) establishment of a first - physical - contact; (ii) a maturation process, during which each connection acquires its characteristic properties; and (iii) a stabilization or elimination phase, during which only robust matches are maintained. In this review, evidence for an astroglial contribution to each of these phases is evaluated (Figure 1). Owing to space limitations, the article focuses mainly on connections in the CNS. However, it should be noted that glial cells also appear to influence synaptogenesis in the peripheral nervous system (PNS) [14-19]).

\section{Astrocytes control synapse number}

The idea that astrocytes play a role in the formation of synaptic contacts arises from a conspicuous temporal correlation between synaptogenesis and the differentiation of this glial cell type [20] (Figure 2). In rodents,

Corresponding author: Frank W. Pfrieger (fw-pfrieger@gmx.de). for example, astrocytes are generated around birth, whereas massive synaptogenesis starts at the end of the first postnatal week and continues for two-to-three weeks $[20,21]$. The long duration of synaptogenesis contrasts with the notion that individual synaptic contacts are formed in less than an hour [22,23]. Evidently, it may simply take weeks to form the incredibly high number of

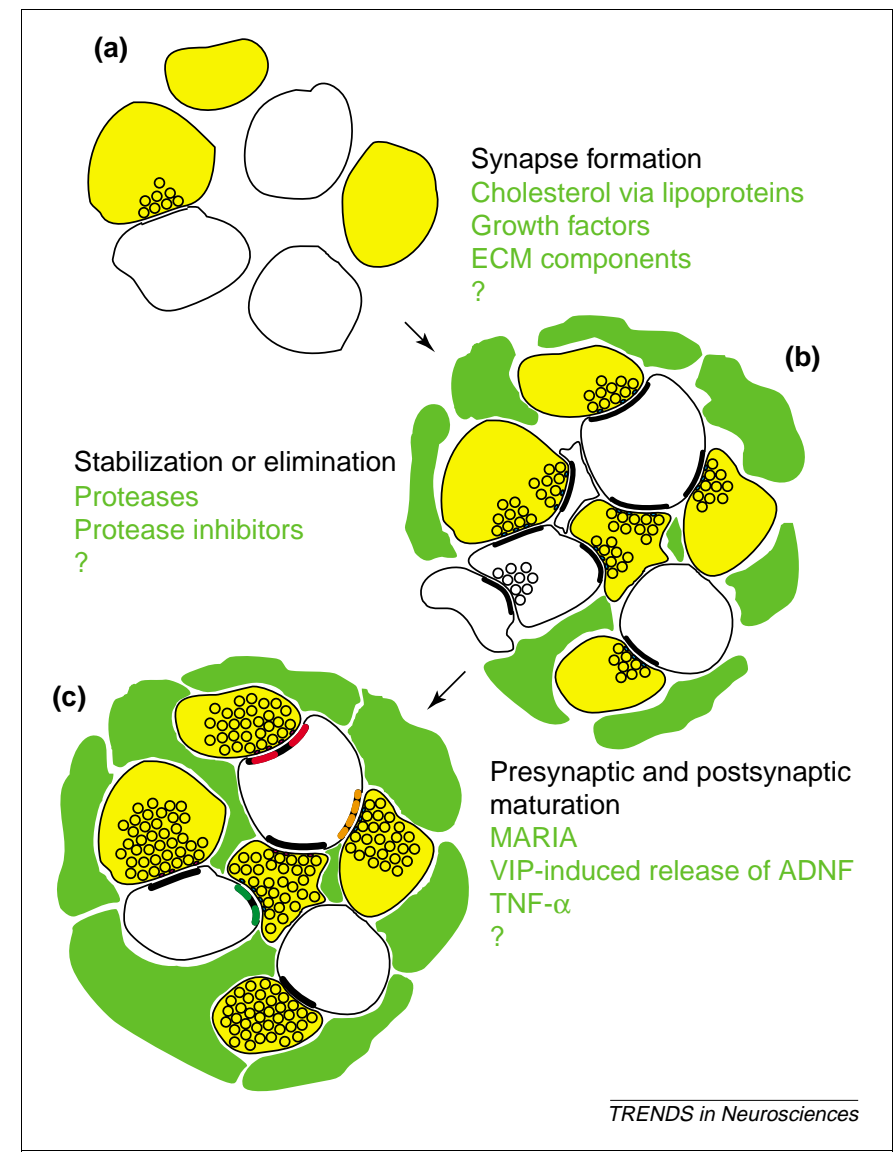

Figure 1. Different stages of synapse development and possible contributions by astrocytes. (a) At the embryonic stage, before astrocytes (green) are generated, neuronal processes [presynaptic (yellow) and postsynaptic (white)] form few and immature synapses. (b) The massive increase in synapse number during the postnatal stage might be enabled by astrocyte-derived components (green text). (c) Excess synapses might be eliminated by astrocytic processes invading the synaptic cleft or by release of proteases that digest synapse-stabilizing components. Astrocyte-derived signals can stabilize surviving synapses and induce presynaptic and postsynaptic maturation processes, including accumulation of vesicles and modifications in the molecular complexes that mediate transmitter release (colored ovals) and reception (thick lines). Question marks indicate that as-yet unidentified factors might also be involved. Abbreviations: ADNF, activitydependent neurotrophic factor; ECM, extracellular matrix; MARIA, muscarinic acetylcholine receptor-inducing activity; TNF- $\alpha$, tumor necrosis factor $\alpha$; VIP, vasoactive intestinal polypeptide. 


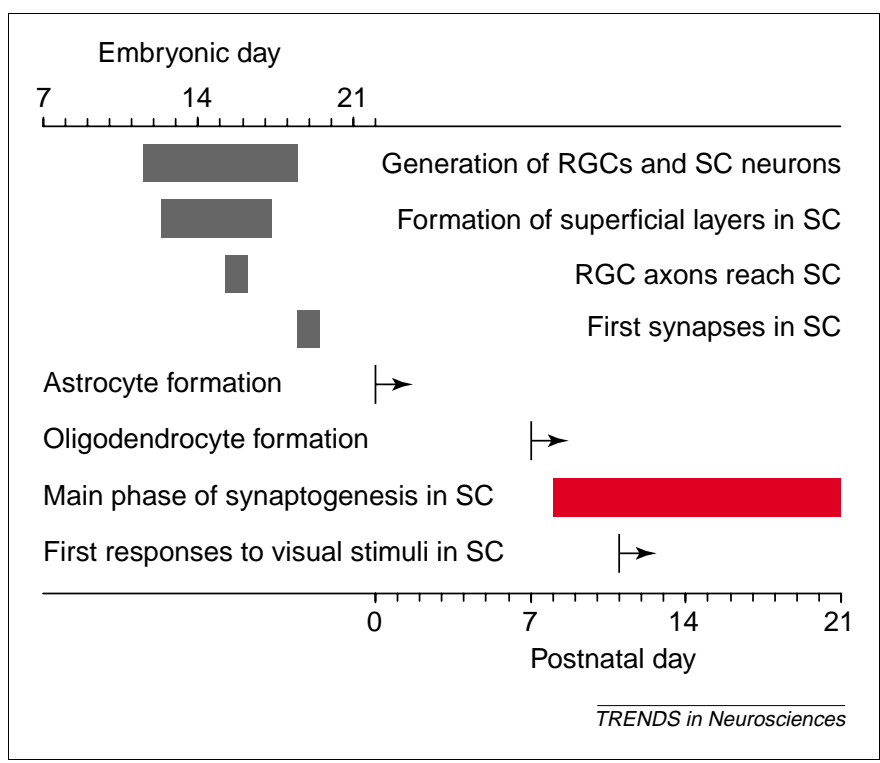

Figure 2. Temporal correlation between synaptogenesis and glial differentiation, exemplified by the rodent retinocollicular pathway. Abbreviations: RGC, retinal ganglion cell; SC, superior colliculus. Reproduced from Ref. [5] by permission of Oxford University Press (http:// www.oup.com).

CNS synapses. Alternatively, the rate of synaptogenesis might be limited by a factor that is provided by astrocytes.

An astrocyte-induced increase in synaptogenesis has been reported in different culture preparations, including neuronal cell lines [24], hippocampal neurons derived from stem cells of adult rats $[25,26]$, and neurons from spinal cord [27], cortex [28], hippocampus [28,29] and hypothalamus [28] of embryonic or perinatal rats. In all of these cases, however, it cannot be excluded that astrocytes increased synapse numbers indirectly by enhancing neuronal survival or axonal and dendritic growth.

Evidence that astrocytes directly enhance synapse formation has been obtained from a series of studies [30-33] on a preparation in which retinal ganglion cells (RGCs) from postnatal rats are highly purified and cultured for two-to-three weeks. The defined, glia-free conditions support high neuronal survival rates and extensive axonal and dendritic growth [34]. The first study showed that cultured RGCs form ultrastructurally defined synapses in the absence of glia [30]. Thus, synapse formation per se appears to be an intrinsic property of these neurons that does not require external signals. However, glia strongly enhanced the level of synaptic activity (Figure 3), and this effect was mimicked by soluble factors released by astrocytes and oligodendrocytes, but not by microglia [30]. Two subsequent studies showed that the glial factor (or factors) enhance the number of synapses severalfold without changing neuronal survival or neurite growth [32,33] (Figure 3). The synaptogenic factor contained in the glia-conditioned medium (GCM) was subsequently identified as cholesterol [31]. This somewhat surprising finding suggests that cholesterol is an astrocyte-derived factor that limits the extent of synaptogenesis [35]. The next steps will be to test this hypothesis in vivo and to clarify how cholesterol promotes synaptogenesis. For example, it could act as a synaptogenic signal after conversion to a steroid or act as building material for
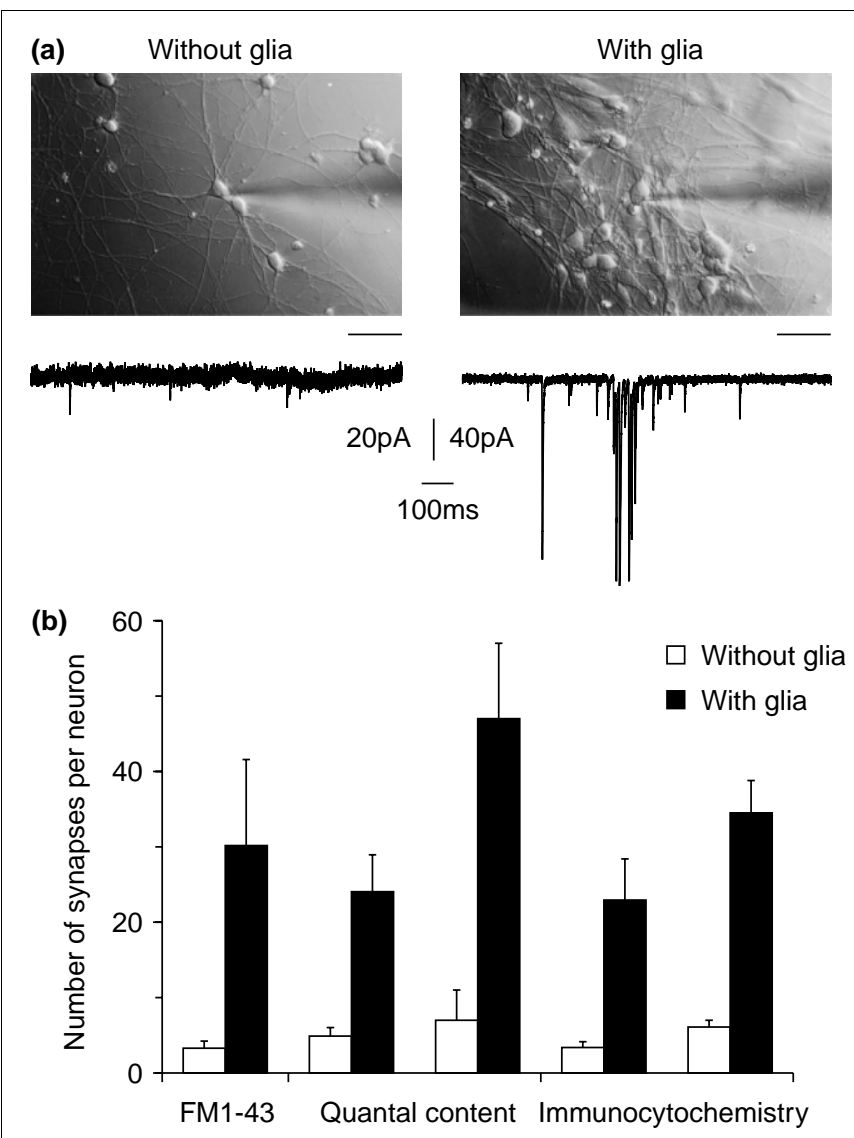

$\overline{T R E N D S ~ i n ~ N e u r o s c i e n c e s ~}$

Figure 3. Glia promote synaptogenesis. (a) Spontaneous synaptic activity in purified retinal ganglion cells (RGCs) that were cultured for $5 \mathrm{~d}$ in defined medium in the absence (left) or presence (right) of collicular glia. Upper panel: Hoffmannmodulation contrast micrographs of RGCs. Scale bar, $50 \mu \mathrm{m}$. Lower panel, traces of whole-cell patch-clamp recordings of spontaneous excitatory postsynaptic currents. Reproduced, with permission, from Ref. [30], (C) (1997) American Association for the Advancement of Science (http://www.sciencemag.org). (b) Glia enhance the number of synapses per neuron, as estimated by three different experimental approaches: FM1-43 labeling of active terminals (using data from Ref. [32]), quantal content of evoked postsynaptic currents (using data from Refs [32,33]) and immunostaining of presynaptic and postsynaptic markers (using data from Refs $[32,33])$. Note that apart from the increase in synapse number, these studies also report glial effects on presynaptic and postsynaptic maturation [32,33].

synaptic components, or it might allow the formation of cholesterol-rich microdomains in membranes, thus enabling signaling processes that are essential for synaptogenesis. There is increasing evidence that presynaptic [36] and postsynaptic [37] components are located in such microdomains [38,39]. In any case, cholesterol is probably not the sole glia-derived factor that influences synapse development. Astrocytes synthesize a variety of components [40-42] and future studies will show which of these affect synapse formation directly without acting on neuronal survival or growth.

\section{Astrocytes help synapses to grow up}

During the maturation phase, synaptic connections acquire their characteristic transmission properties. This involves changes in the number of vesicles in presynaptic terminals, and changes in the composition of the exocytosis machinery and the postsynaptic receptor complex $[9,43,44]$ (Figure 1). The studies on purified RGCs 
mentioned in the preceding section showed that glial cells enhance the size of miniature postsynaptic currents $[30,32,33]$ and that this effect is mediated, at least in part, by a direct increase in postsynaptic transmitter sensitivity [33]. Furthermore, the glia-induced changes develop with a delay of at least $24 \mathrm{~h}$, which excludes fast modulatory actions [32].

Cholesterol may not mimic all of these glial effects; other factors, including membrane-delimited signals [32], could also control synaptic maturation. Recently, several such factors have appeared on the scene. Beattie et al. [45] showed that glia-derived tumor necrosis factor $\alpha(\mathrm{TNF}-\alpha)$ raises the surface expression of glutamate receptors in hippocampal neurons from postnatal rats in vitro and in acute slices. However, application of TNF- $\alpha$ induced a rapid increase (within minutes) of the glutamate-receptor density at synapses, respectively. This fast action implies a modulatory function of TNF- $\alpha$ and excludes the possibility that it mediates the slow GCM-induced increase in quantal size in RGC cultures [32]. Another candidate for a glia-derived maturation signal is activity-dependent neurotrophic factor (ADNF), which is released from astrocytes in response to neuron-derived vasoactive intestinal polypeptide [46]. In turn, ADNF enhances the synaptic connectivity and density of postsynaptic NMDA receptors in cultured hippocampal neurons from embryonic rats, either directly or by stimulating the release of neurotrophins [47]. A glia-derived signal that controls the expression of specific transmitter receptors has been detected in chick retina [48]. Cultured Müller glia secrete an as-yet unidentified protein termed muscarinic-acetylcholine-receptor-inducing activity (MARIA), which induces the expression of a specific subtype (M2) of muscarinic receptors in retinal neurons of chick embryos in ovo. This effect could help to explain why during retinal development the M2 receptor appears following differentiation of Müller glia.

Finally, there appears to be a link between astrocytes and the most prominent synaptogenic factor, agrin, a motoneuron-derived signal that is essential for the formation of neuromuscular junctions (NMJs) [18] and that might play a role in synaptogenesis in the CNS [49]. Contact with mouse glia reduced levels of mRNA encoding agrin in cultured rat hippocampal neurons, whereas soluble glial factors halved the expression of a specific isoform but left the total level unaffected [50]. Notably, an interesting study showed recently that neurotrophins support survival and growth of cultured frog spinal cord neurons, but inhibit agrin synthesis and thus NMJ formation. However, soluble, as-yet unidentified Schwann-cell-derived factors override this inhibition and switch motoneurons to a synaptogenic state [16]. It will be interesting to see whether such a binary regulation of NMJ formation is implemented in vivo and whether a similar principle applies to synaptogenesis in the CNS.

Do astrocytes enhance presynaptic maturation? Nägler et al. [32] showed directly that GCM enhances the efficacy of transmitter release from individual terminals in cultured RGCs. Cholesterol completely mimicked the increase in the frequency of asynchronous transmitter release [31], suggesting that cholesterol raises the release capacity of synapses, possibly by enhancing vesicle biogenesis or the efficacy of the release apparatus [38]. During maturation, synaptic terminals acquire a characteristic set of voltage-activated $\mathrm{Ca}^{2+}$ channels that mediate actionpotential-evoked transmitter release [51]. Astrocyte-derived signals might affect this process in a similar way to oligodendrocytes or Schwann cells, which guide ion-channel clustering at nodes of Ranvier [52-54]. Indeed, there is evidence that contact with astrocytes enhances the density of specific subtypes of whole-cell $\mathrm{Ca}^{2+}$ currents in hippocampal neurons in vitro [27,55], although it remains unclear which signals mediate these changes and whether they also control the presynaptic pool of channels.

The reports summarized here suggest that signals from astrocytes regulate specific aspects of presynaptic and postsynaptic maturation, including receptor density and subunit composition, as well as the efficacy of transmitter release (Figure 1). It remains to be seen whether these signals control synapse development in vivo. Notably, there is very little information on the reverse effect - that synapses regulate the differentiation of surrounding astrocytic processes. If synapses are indeed tripartite and include astrocytes as supporting and modulatory elements, then the functional properties of astrocytic processes must match those of the synapses that they ensheath [56]. This matching process is probably mediated by astrocyte- and neuron-derived signals that regulate the functional maturation of synapses and their astrocytic sheathes, respectively. Accordingly, there is evidence that neurons regulate the expression of specific glutamate transporters in astrocytes [57].

\section{Astrocytes determine the fate of synapses}

During brain development, only some of the newly formed synapses are strengthened and maintained, whereas all others are eliminated $[9,18,58]$ (Figure 1). Do astrocytes help to maintain synapses? There is little experimental evidence for such an effect. Ablation of astrocytes in vivo causes loss of connections [59] but this could be a consequence, rather than the cause, of massive neurodegeneration. Barres and colleagues [33] reported that removal of astrocytic feeding layers from cultured RGCs leads to a decrease in synapse number, suggesting that soluble glial signals are necessary for synapse maintenance. However, this effect could have been caused indirectly by degeneration of axons and dendrites. Interestingly, a recent study on mouse hippocampal slices suggests that astrocytic processes determine the stability and mobility of dendritic spines via ephrinreceptor activation [60] which, in turn, could affect synapse turnover.

Do astrocytes exert the opposite effect and actively eliminate synapses? A first clue came from a study on explant cerebellar cultures in which the number of astrocytes or granule cells had been pharmacologically reduced. Addition of astrocytes to these cultures reduced the density of somatic synapses [61]. Evidence that Bergmann glia regulate climbing fiber innervation of Purkinje cells came from a study on transgenic animals in which the $\mathrm{Ca}^{2+}$ conductance of glutamate receptors in Bergmann glia could be manipulated in a temporally 
controlled manner [62]. Experimentally induced retraction of Bergmann glia processes from Purkinje cells in three-week-old rats in vivo left a quarter of the neurons innervated by multiple climbing fibers [62]. Notably, Purkinje cells had already attained monosynaptic innervation before glial retraction was induced, suggesting that removal of the glial sheath allowed reinnervation. It remains to be seen whether removal of glial processes at an earlier stage prevents the elimination of climbing fiber inputs. Sims and Gilmore [63] showed that irradiation of the spinal cord in newborn rats induces invasion of Schwann cells and loss of synapses in spinal cord gray matter, suggesting that Schwann cells eliminate synapses. However, it is not clear whether synapses were eliminated by invading glial processes or by the expulsion of astrocytes. Finally, hormone- and activity-dependent synapse elimination occurs in different brain areas in adults. That these changes are accompanied by extension and retraction of astrocytic processes supports the idea that astrocytes play an active role in synapse elimination [4,64-67]. How can astrocytes eliminate synapses? They could remove them mechanically by invading the synaptic cleft and 'eating up' terminals and spines. Alternatively, astrocytes could destabilize components that guarantee the stability of synapses, for example by releasing proteolytic enzymes (Figure 1). A recent study has shown that matrix metalloproteinase-3, which is produced by glial cells, can remove agrin from the basal lamina of NMJs [68].

Taken together, the current literature suggests several hypotheses about the role of astrocytes in synapse maintenance and elimination. Further experiments will be required to identify the signaling pathways involved and to determine their relevance in vivo.

\section{Concluding remarks}

Astrocytes possibly contribute to several stages of synapse development in the CNS. The hypotheses presented here rely mainly on studies using culture preparations; the molecular mechanisms of these interactions are still largely unknown. Progress in this area depends on three issues. First, the astrocyte-derived signals that control synapse development must be identified. This requires new preparations, where neurons from the postnatal CNS can be studied in the absence and presence of glial cells. Second, the different hypotheses must be tested in vivo. This requires new target genes and new animal models, so that astrocytes can be genetically manipulated in a temporally controlled way. Important contributions to these aspects could also come from studies on genetically tractable organisms such as Drosophila [69]. Finally, we need to address the still-neglected question of whether and how the different types of synapses control the functional specialization of the astrocytic processes that surround them. A reciprocal control of differentiation appears indispensable for astrocytes to support and modulate synapse function. Notably, the large diversity of synapses in the CNS implies that not all synapses are created equal: some of them might require astrocytic signals for their development, whereas others might not. In any case, the current state of affairs signifies that a full understanding of synaptogenesis during development, plasticity and regeneration is not possible without taking astrocytes into account.

\section{Acknowledgements}

Research in our laboratory is supported by the Centre Nationale de la Recherche Scientifique, the Max-Planck-Gesellschaft, the Fondation Pour La Recherche Medicale, the Fondation Electricite de France, the Ara Parseghian Medical Research Foundation, the Region Alsace, and the Deutsche Forschungsgemeinschaft.

\section{References}

1 Araque, A. et al. (2001) Dynamic signaling between astrocytes and neurons. Annu. Rev. Physiol. 63, 795-813

2 Castonguay, A. et al. (2001) Glial cells as active partners in synaptic functions. Prog. Brain Res. 132, 227-240

3 Fields, R.D. and Stevens-Graham, B. (2002) New insights into neuron-glia communication. Science 298, 556-562

4 Oliet, S.H. (2002) Functional consequences of morphological neuroglial changes in the magnocellular nuclei of the hypothalamus. J. Neuroendocrinol. 14, 241-246

5 Volterra, A., et al. eds (2002) The Tripartite Synapse: Glia In Synaptic Transmission, Oxford University Press

6 Araque, A. et al. (1999) Tripartite synapses: glia, the unacknowledged partner. Trends Neurosci. 22, 208-215

7 Umeda, T. and Okabe, S. (2001) Visualizing synapse formation and remodeling: recent advances in real-time imaging of CNS synapses. Neurosci. Res. 40, 291-300

8 Garner, C.C. et al. (2002) Molecular mechanisms of CNS synaptogenesis. Trends Neurosci. 25, 243-251

9 Cohen-Cory, S. (2002) The developing synapse: construction and modulation of synaptic structures and circuits. Science 298, 770-776

10 Jin, Y. (2002) Synaptogenesis: insights from worm and fly. Curr. Opin. Neurobiol. 12, 71-79

11 Zito, K. and Svoboda, K. (2002) Activity-dependent synaptogenesis in the adult mammalian cortex. Neuron 35, 1015-1017

12 Packard, M. et al. (2003) Wnts and TGF $\beta$ in synaptogenesis: old friends signalling at new places. Nat. Rev. Neurosci. 4, 113-120

13 Scheiffele, P. Cell-cell signaling during synapse formation in the CNS. Annu. Rev. Neurosci. (in press)

14 Macleod, G.T. et al. (2001) Formation and function of synapses with respect to Schwann cells at the end of motor nerve terminal branches on mature amphibian (Bufo marinus) muscle. J. Neurosci. 21, 2380-2392

15 Yang, J.F. et al. (2001) Schwann cells express active agrin and enhance aggregation of acetylcholine receptors on muscle fibers. J. Neurosci. 21, 9572-9584

16 Peng, H.B. et al. (2003) Differential effects of neurotrophins and Schwann cell-derived signals on neuronal survival/growth and synaptogenesis. J. Neurosci. 23, 5050-5060

17 Son, Y.J. et al. (1996) Schwann cells induce and guide sprouting and reinnervation of neuromuscular junctions. Trends Neurosci. 19, $280-285$

18 Sanes, J.R. and Lichtman, J.W. (1999) Development of the vertebrate neuromuscular junction. Annu. Rev. Neurosci. 22, 389-442

19 Auld, D.S. and Robitaille, R. (2003) Perisynaptic Schwann cells at the neuromuscular junction: nerve- and activity-dependent contributions to synaptic efficacy, plasticity, and reinnervation. Neuroscientist 9, 144-157

20 Pfrieger, F.W. and Barres, B.A. (1996) New views on synapse-glia interactions. Curr. Opin. Neurobiol. 6, 615-621

21 Sauvageot, C.M. and Stiles, C.D. (2002) Molecular mechanisms controlling cortical gliogenesis. Curr. Opin. Neurobiol. 12, 244-249

22 Friedman, H.V. et al. (2000) Assembly of new individual excitatory synapses: time course and temporal order of synaptic molecule recruitment. Neuron 27, 57-69

23 Gomperts, S.N. et al. (2000) Distinct roles for ionotropic and metabotropic glutamate receptors in the maturation of excitatory synapses. J. Neurosci. 20, 2229-2237

24 Hartley, R.S. et al. (1999) Functional synapses are formed between human NTera2 (NT2N, hNT) neurons grown on astrocytes. J. Comp. Neurol. 407, 1-10 
25 Toda, H. et al. (2000) Neurons generated from adult rat hippocampal stem cells form functional glutamatergic and GABAergic synapses in vitro. Exp. Neurol. $165,66-76$

26 Song, H.J. et al. (2002) Neural stem cells from adult hippocampus develop essential properties of functional CNS neurons. Nat. Neurosci. $5,438-445$

$27 \mathrm{Li}$, Y.X. et al. (1999) Astrocytes regulate the developmental appearance of GABAergic and glutamatergic postsynaptic currents in cultured embryonic rat spinal neurons. Eur. J. Neurosci. 11, 2537-2551

28 van den Pol, A.N. and Spencer, D.D. (2000) Differential neurite growth on astrocyte substrates: interspecies facilitation in green fluorescent protein-transfected rat and human neurons. Neuroscience 95, $603-616$

29 Verderio, C. et al. (1999) Astrocytes are required for the oscillatory activity in cultured hippocampal neurons. Eur. J. Neurosci. 11, $2793-2800$

30 Pfrieger, F.W. and Barres, B.A. (1997) Synaptic efficacy enhanced by glial cells. Science $277,1684-1687$

31 Mauch, D.H. et al. (2001) CNS synaptogenesis promoted by gliaderived cholesterol. Science 294, 1354-1357

32 Nägler, K. et al. (2001) Glia-derived signals induce synapse formation in neurones of the rat central nervous system. J. Physiol. 533, 665-679

33 Ullian, E.M. et al. (2001) Control of synapse number by glia. Science $291,657-661$

34 Göritz, C. et al. (2002) Role of glia-derived cholesterol in synaptogenesis: new revelations in the synapse-glia affair. J. Physiol. (Paris) 96 , $257-263$

35 Pfrieger, F.W. (2003) Outsourcing in the brain: do neurons depend on cholesterol delivery by astrocytes? BioEssays 25, 72-78

36 Mitter, D. et al. (2003) The synaptophysin/synaptobrevin interaction critically depends on the cholesterol content. J. Neurochem. 84, 35-42

37 Hering, H. et al. (2003) Lipid rafts in the maintenance of synapses, dendritic spines, and surface AMPA receptor stability. J. Neurosci. 23, $3262-3271$

38 Pfrieger, F.W. (2003) Role of cholesterol in synapse formation and function. Biochim. Biophys. Acta 1610, 271-280

39 Tsui-Pierchala, B.A. et al. (2002) Lipid rafts in neuronal signaling and function. Trends Neurosci. 25, 412-417

40 Lafon-Cazal, M. et al. (2003) Proteomic analysis of astrocytic secretion in the mouse. Comparison with the cerebrospinal fluid proteome. J. Biol. Chem. 278, 24438-24448

41 Gomes, F.C. et al. (2001) Cross-talk between neurons and glia: highlights on soluble factors. Braz. J. Med. Biol. Res. 34, 611-620

$42 \mathrm{Du}$, Y.Z. and Dreyfus, C.F. (2002) Oligodendrocytes as providers of growth factors. J. Neurosci. Res. 68, 647-654

43 Fitzsimonds, R.M. and Poo, M.M. (1998) Retrograde signaling in the development and modification of synapses. Physiol. Rev. 78, 143-170

44 Atwood, H.L. and Karunanithi, S. (2002) Diversification of synaptic strength: presynaptic elements. Nat. Rev. Neurosci. 3, 497-516

45 Beattie, E.C. et al. (2002) Control of synaptic strength by glial TNF $\alpha$. Science 295, 2282-2285

46 Gozes, I. and Brenneman, D.E. (2000) A new concept in the pharmacology of neuroprotection. J. Mol. Neurosci. 14, 61-68

47 Blondel, O. et al. (2000) A glia-derived signal regulating neuronal differentiation. J. Neurosci. 20, 8012-8020
48 Belmonte, K.E. et al. (2000) Developmental expression of muscarinic acetylcholine receptors in chick retina: selective induction of M2 muscarinic receptor expression in ovo by a factor secreted by Muller glial cells. J. Neurosci. 20, 8417-8425

49 Böse, C.M. et al. (2000) Agrin controls synaptic differentiation in hippocampal neurons. J. Neurosci. 20, 9086-9095

50 Lesuisse, C. et al. (2000) Regulation of agrin expression in hippocampal neurons by cell contact and electrical activity. Brain Res. Mol. Brain Res. 81, 92-100

51 Meir, A. et al. (1999) Ion channels in presynaptic nerve terminals and control of transmitter release. Physiol. Rev. 79, 1019-1088

52 Pedraza, L. et al. (2001) Organizing principles of the axoglial apparatus. Neuron $30,335-344$

53 Rasband, M.N. and Trimmer, J.S. (2001) Developmental clustering of ion channels at and near the node of Ranvier. Dev. Biol. 236, 5-16

54 Girault, J.A. and Peles, E. (2002) Development of nodes of Ranvier. Curr. Opin. Neurobiol. 12, 476-485

55 Mazzanti, M. and Haydon, P.G. (2003) Astrocytes selectively enhance N-type calcium current in hippocampal neurons. Glia 41, 128-136

56 Riquelme, R. et al. (2002) Bergmann glia $\mathrm{GABA}_{\mathrm{A}}$ receptors concentrate on the glial processes that wrap inhibitory synapses. J. Neurosci. 22, 10720-10730

57 Danbolt, N.C. (2001) Glutamate uptake. Prog. Neurobiol. 65, 1-105

58 Personius, K.E. and Balice-Gordon, R.J. (2000) Activity-dependent editing of neuromuscular synaptic connections. Brain Res. Bull. 53, $513-522$

59 Cui, W. et al. (2001) Inducible ablation of astrocytes shows that these cells are required for neuronal survival in the adult brain. Glia 34, $272-282$

60 Murai, K.K. et al. (2003) Control of hippocampal dendritic spine morphology through ephrin-A3/EphA4 signaling. Nat. Neurosci. 6, $153-160$

61 Seil, F.J. (2001) Interactions between cerebellar Purkinje cells and their associated astrocytes. Histol. Histopathol. 16, 955-968

62 Iino, M. et al. (2001) Glia-synapse interaction through $\mathrm{Ca}^{2+}$. permeable AMPA receptors in Bergmann glia. Science 292, 926-929

63 Sims, T.J. and Gilmore, S.A. (2000) Schwann cell-induced loss of synapses in the central nervous system. Brain Res. 882, 221-225

64 Hatton, G.I. (1997) Function-related plasticity in hypothalamus. Annu. Rev. Neurosci. 20, 375-397

65 Garcia-Segura, L.M. et al. (1999) Role of astroglia in estrogen regulation of synaptic plasticity and brain repair. J. Neurobiol. 40, 574-584

66 Mong, J.A. and McCarthy, M.M. (1999) Steroid-induced developmental plasticity in hypothalamic astrocytes: implications for synaptic patterning. J. Neurobiol. 40, 602-619

67 Theodosis, D.T. and Poulain, D.A. (2001) Maternity leads to morphological synaptic plasticity in the oxytocin system. Prog. Brain Res. 133, 49-58

68 VanSaun, M. and Werle, M.J. (2000) Matrix metalloproteinase-3 removes agrin from synaptic basal lamina. J. Neurobiol. 43, 140-149

69 Freeman, M.R. et al. (2003) Unwrapping glial biology: Gcm target genes regulating glial development, diversification, and function. Neuron $38,567-580$

\section{Managing your references and BioMedNet Reviews}

Did you know that you can now download selected search results from BioMedNet Reviews directly into your chosen referencemanaging software? After performing a search, simply click to select the articles you are interested in, choose the format required (e.g. EndNote 3.1) and the bibliographic details, abstract and link to the full-text will download into your desktop reference manager database.

BioMedNet Reviews is available on institute-wide subscription. If you do not have access to the full-text articles in BioMedNet Reviews, ask your librarian to contact reviews.subscribe@biomednet.com 